\title{
Manual Annotation Studio (MAS): a collaborative platform for manual functional annotation of viral and microbial genomes
}

Matthew R. Lueder ${ }^{1,2}$, Regina Z. Cer', Miles Patrick ${ }^{1}$, Logan J. Voegtly ${ }^{1,2}$, Kyle A. Long ${ }^{1,2}$, Gregory K. Rice ${ }^{1,2}$ and Kimberly A. Bishop-Lilly ${ }^{1 *}$ (D)

\begin{abstract}
Background: Functional genome annotation is the process of labelling functional genomic regions with descriptive information. Manual curation can produce higher quality genome annotations than fully automated methods. Manual annotation efforts are time-consuming and complex; however, software can help reduce these drawbacks.

Results: We created Manual Annotation Studio (MAS) to improve the efficiency of the process of manual functional annotation prokaryotic and viral genomes. MAS allows users to upload unannotated genomes, provides an interface to edit and upload annotations, tracks annotation history and progress, and saves data to a relational database. MAS provides users with pertinent information through a simple point and click interface to execute and visualize results for multiple homology search tools (blastp, rpsblast, and HHsearch) against multiple databases (Swiss-Prot, nr, CDD, PDB, and an internally generated database). MAS was designed to accept connections over the local area network (LAN) of a lab or organization so multiple users can access it simultaneously. MAS can take advantage of high-performance computing (HPC) clusters by interfacing with SGE or SLURM and data can be exported from MAS in a variety of formats (FASTA, GenBank, GFF, and excel).

Conclusions: MAS streamlines and provides structure to manual functional annotation projects. MAS enhances the ability of users to generate, interpret, and compare results from multiple tools. The structure that MAS provides can improve project organization and reduce annotation errors. MAS is ideal for team-based annotation projects because it facilitates collaboration.
\end{abstract}

Keywords: Genome annotation, Gene annotation, Functional annotation, Manual annotation, Phage annotation, Bioinformatics, High-performance computing, Phage, Microbial genomics

\section{Background}

Genome annotation is the process of locating and labelling functional regions within a genome. The location of functional regions is determined in a process called gene

\footnotetext{
* Correspondence: Kimberly.a.bishop-lilly.civ@mail.mil

${ }^{1}$ Genomics and Bioinformatics Department, Biological Defense Research Directorate, Naval Medical Research Center, Fort Detrick, MD, USA Full list of author information is available at the end of the article
}

calling. Typically, this is performed using automated software tools such as Glimmer [1], GeneMark [2], or Prodigal [3] which use statistical modelling to predict the presence and location of each gene. After gene calling, a descriptive label and metadata are provided for each predicted gene in a process called functional annotation. A fundamental use of annotation is to discover and characterize genes of interest. For example, human

C C The Author(s). 2021 Open Access This article is licensed under a Creative Commons Attribution 4.0 International License, which permits use, sharing, adaptation, distribution and reproduction in any medium or format, as long as you give appropriate credit to the original author(s) and the source, provide a link to the Creative Commons licence, and indicate if changes were made. The images or other third party material in this article are included in the article's Creative Commons licence, unless indicated otherwise in a credit line to the material. If material is not included in the article's Creative Commons licence and your intended use is not permitted by statutory regulation or exceeds the permitted use, you will need to obtain permission directly from the copyright holder. To view a copy of this licence, visit http://creativecommons.org/licenses/by/4.0/ The Creative Commons Public Domain Dedication waiver (http://creativecommons.org/publicdomain/zero/1.0/) applies to the data made available in this article, unless otherwise stated in a credit line to the data. 
genome annotation may be used for discovering diseasecausing variants [4], prokaryotic annotation for discovering genes responsible for pathogenicity or antibiotic resistance [5], and viral genome annotation for discovering potential targets for therapeutics [6]. There have been several efforts to completely automate the process of microbial genome annotation; Systems such as RAST (Rapid Annotation using Subsystems Technology) [7], Prokka [8], and the Prokaryotic Genome Annotation Pipeline (PGAP) [9] can predict gene locations and provide a functional annotation with the click of a button. The previously described annotation use cases require detailed and accurate annotations. While these automated tools provide convenience, manual curation is needed for more accurate and detailed annotations $[10,11]$.

Optimal manual functional annotation involves using an ensemble approach, where multiple tools and databases are used and results from each are compared and contrasted [11]. There are many examples in the literature which use this approach to improve upon previous annotations [12-15]. This approach is typically accomplished by submitting separate searches to multiple disparate web servers and comparing results by clicking between tabs then saving the final annotation in a text file or spreadsheet. With this method, search results are transient, there is significant overhead to start the searches, and direct comparison of results is more difficult.

To improve this process, we developed Manual Annotation Studio (MAS), a software tool that assists users throughout the process of manual functional annotation. MAS is a user-deployable web server that provides an interface for creating and editing annotations and collecting and reviewing evidence for the function of genes. MAS allows users to perform an ensemble of homology searches at the click of a button and provides interactive visualizations of search results and of the genome being annotated. It organizes annotations into a structured database, allowing them to be easily searched. It also facilitates team efforts by allowing multiple users to work on the same data concurrently, and encourages consistency of annotations by automatically generating a searchable BLAST database of previously annotated proteins. MAS can be run offline and can utilize highperformance computing (HPC) clusters through SGE (Oracle Grid Engine) or SLURM (Simple Linux Utility for Resource Management).

\section{Results and discussion}

\section{Comparison to existing tools}

Current commonly used annotation editing tools include GenDB [16], Apollo [17], DNA Master [18], and CLC Genomics Workbench. Here, we define an annotation editing tool as any tool which allows users to import a genomic sequence, to manually label regions within the genomic sequence with functional information, and to export the fully annotated sequence.

GenDB is an annotation editing tool which allows users to view results for multiple functional annotation tools. GenDB supports the annotation of prokaryotic genomes and more recently, the annotation of eukaryotic genomes through GenDBE. Similar to MAS, it performs automated gene calling, supports multiple functional annotation tools, and saves annotations and tool results to a database. In addition, GenDB performs automated gene calling. GenDB was originally created by Bielefeld University as an open source alternative to the commercial and/or closed source annotation tools. However, GenDB is currently maintained by the University of Giessen and it is no longer open source. GenDB is offered as a web service hosted on infrastructure provided by the University of Giessen and accessed by users through a web browser. To get an account to use GenDB, users agree to either an academic or a commercial partnership with the university. The university will then upload genomes to the server on behalf of the user, run the gene calling and functional annotation tools, and backup user data. In contrast, MAS is fully open source, allows users to keep their data completely private, and can be used without intervention from an outside group. However, MAS requires users to install and run it on their own infrastructure.

Apollo is an open-source annotation editing platform. Like MAS, it supports collaboration and can be installed locally with Docker. However, Apollo focuses on gene structure annotation rather than functional annotation. Gene structure annotation is the process of identifying the location of genes, alternative splicing sites (exons and introns), and regulatory sequences. MAS does not focus on this aspect of annotation and instead predicts gene coordinates with automated gene-callers or lets users upload custom coordinates. Functional annotation within Apollo is performed by exporting protein sequences as FASTA files and using tools outside of Apollo to find homology. Because MAS and Apollo focus on different aspects of annotation, these tools can be used in conjunction. In situations where automated gene calling is not accurate enough, Apollo can be used to determine the coordinates of coding sequences, these coordinates can be imported into MAS, and MAS can be used to functionally annotate the genome.

DNA Master is a genome annotation tool that has become a popular option for bacteriophage genome annotation because of its use in the SEA-PHAGES project [19]. It is available as an executable for Windows machines and can be used for structural and functional annotation. DNA master allows users to adjust the coordinates of coding sequences based on the results of 
multiple gene callers and evidence from sequence homology. DNA Master provides a form for editing functional annotations and is integrated with NCBI's BLAST API, which provides results from BLAST searches against NCBI's non-redundant protein (nr) database. However, functional annotations based on a single tool and database are not as accurate or descriptive as functional annotations based on evidence from multiple sources. DNA Master runs locally, on a single machine, and does not provide a framework for collaborative annotation. As with Apollo, CDS coordinates which have been refined using DNA Master's manual structural annotation capabilities can be imported into MAS.

CLC Genomics workbench supports a wide array of genomic analyses, including manual genome annotation. It supports basic automated gene calling and also allows users to add annotations to manually selected genomic regions. The integration of BLAST and HMMER provides support for functional annotation. However, CLC Genomics Workbench is a commercial software product that must be purchased to use.

\section{Genomes, features, and annotations}

MAS manages three main types of data: genomes, features, and annotations. This data is created when a user uploads a genomic sequence to MAS. Each genome in MAS contains the genomic sequence of an organism and is associated with a number of features. A feature, on the other hand, is specific to a single genome and describes the location of a coding sequence (CDS), tRNA, or repeat region. Further information about a feature is contained within its associated annotation. An annotation contains an accession, a label, a sequence, notes fields, a flag, and an optional user assignment. Accessions are assigned to annotations automatically while labels, notes fields, and flags are assigned during the manual annotation process. The sequence of an annotation is unique to it (i.e. only one annotation exists per unique protein) and can be a protein sequence, an RNA sequence, or a DNA sequence depending on the type of feature with which it is associated. Two features will be associated with the same annotation if they have identical sequences. For example, if two phage genomes are uploaded and they both encode a protein with an identical sequence, then each phage will have their own feature but will share an annotation.

\section{Genome upload}

Genome sequences can be provided to MAS as a singlesequence FASTA file. Upon upload, a genome and associated features and annotations will be created and saved to the MAS database. Genome sequences can be uploaded through the phage genome upload, bacterial genome upload, or custom upload tabs. If a genome is uploaded through the phage or bacterial genome upload tabs, gene-calling is automated with Glimmer (v3.02) to find CDSs and tRNAscan-se (v2.0.6) to find tRNA genes. When uploading a phage genome, users can choose to re-orient the genome from the terminase gene. Using this option is a best practice when genomic termini cannot be determined based on packaging strategy and ensures similar genomes start from the same position, facilitating comparative genomics [20]. If this option is selected, MAS will automatically search all CDSs using blastp against a database of terminase proteins. If there is enough evidence of a terminase gene, it will automatically re-orient the genome based on that genomic position. Whereas if the genome contains both small and large terminase subunits, the genome will be re-oriented to start from whatever subunit is upstream. If the phage genome contains direct terminal repeats, the user can enter the size of the repeats and features will be created to represent them. The custom upload tab allows users to upload their own gene-calls. When uploading a genome sequence, the user has the option to assign all new annotations created as a result of the upload to a specific user.

\section{The result viewer}

The result viewer can be accessed through one of the result navigation options or through links in the genome and annotation lists and details views. This is where homology searches are launched, annotations are edited, and search results are visualized. One annotation is displayed at a time but the navigation pane allows users to switch to different annotations easily (Fig. 1A). The function of the navigation pane depends on the selected navigation option. There are three navigation options: navigate by flag, navigate by assignment, and navigate by genome. If the navigate by flag option is being used, the navigation pane will guide the user through annotations assigned to the selected flag option, sorted by accession. If the navigate by assignment option is being used, the user will be able to navigate through all annotations assigned to a specific assigned user, also sorted by accession. Finally, if the navigate by genome option is selected, the navigation pane can be used to navigate through annotations associated with the selected genome, sorted by their genomic coordinates. If the selected genome has fewer than 1000 features, an interactive visualization of the genome will appear in the navigation pane (Fig. 1B). This visualization can be used to navigate through the annotations assigned to the selected genome.

Homology searches can be launched on an individual basis or, if the navigate by genome option is being used, for all proteins in a specific genome. Individual searches are initiated with the 'Run' button in the result visualization pane (Fig. 1E). The tool to use and the 

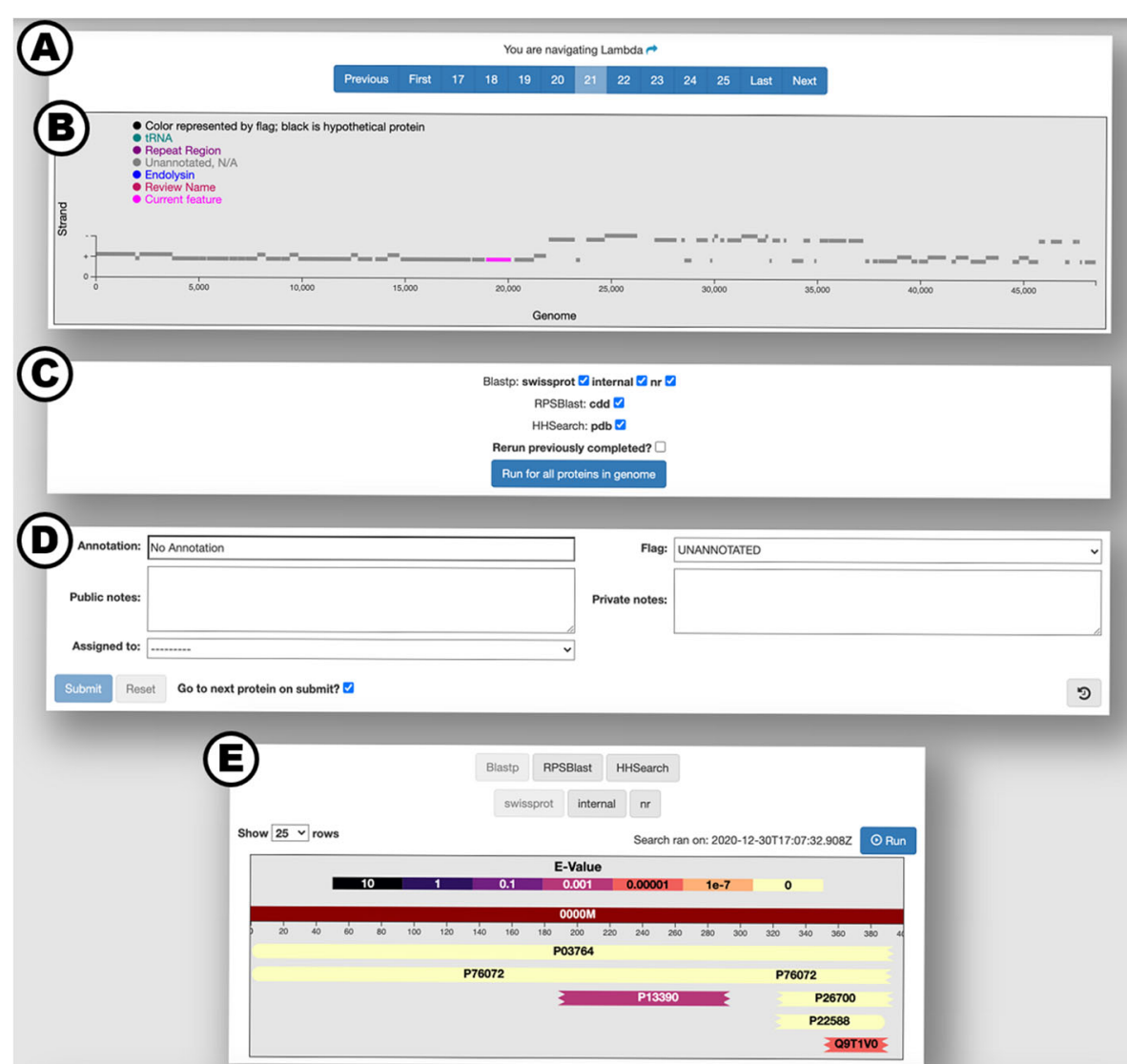

(F)

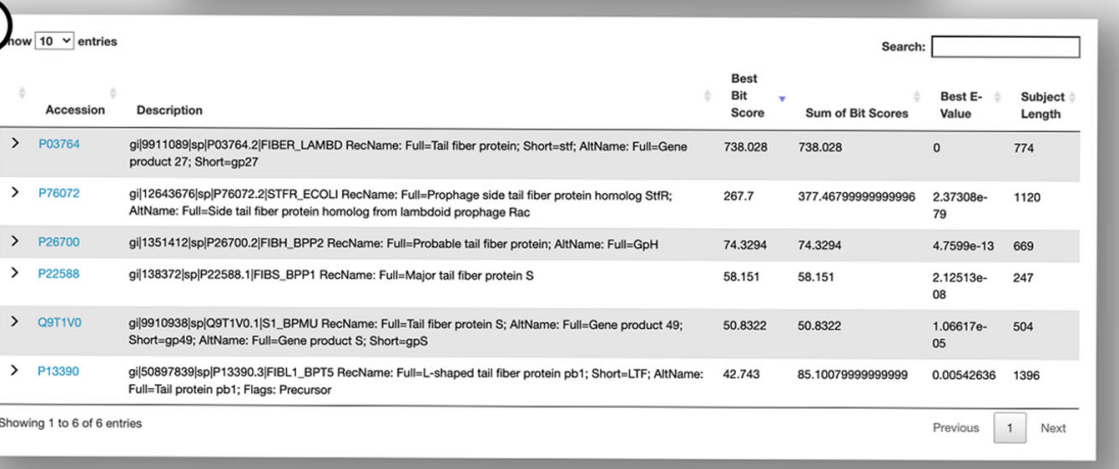

Fig. 1 The result viewer page with the 'navigate by genome' navigation option selected. A The navigation pane gives the user the ability to navigate through the selected group of Annotations. Here, CDS 21 of phage lambda is selected. B The genome visualization depicts each feature in the selected genome at its genomic coordinates. The color of the feature is determined by the flag assigned to the annotation associated with the feature (e.g. blue for endolysin, purple for repeat region). Hovering over a feature will display information about the feature's annotation and clicking it will navigate to that annotation. $\mathbf{C}$ When the 'navigate by genome' option is selected, users have the ability to initiate homology searches for all proteins in the selected genome. D The annotation form allows users to edit the attributes of an annotation. The history of an annotation can be displayed by clicking the "show history" button in the bottom right. E The result visualization pane allows users to select which tool and database for which they would like to view results. Proteins/domains returned from the selected search are shown as colored blocks aligned against the query sequence. The color of the block represents the statistical significance of the alignment (e.g. black for e-value 10 to light yellow for e-value 0). Clicking on a block highlights the corresponding result in the result table. Single searches are initiated from this pane. $\mathbf{F}$ The result table contains additional information for each hit returned by the selected homology search. The name and length of the returned subject/target are given, along with additional statistical metrics. Users can see the entire alignment in text format using the drop-down arrows. Links are available to navigate to further information about the subject/target in its native database 
database to search against can be selected with the buttons at the top of the navigation pane. If the navigate by genome option is used, there will be an additional pane below the navigation pane which will allow users to select tool/database combinations for searching every protein in the genome (Fig. 1C).

After search results are returned to the server, they can be viewed in the result visualization and result table. The result visualization depicts how each protein returned from the database aligns to the query, showing where the alignment starts and stops on the query and the statistical significance of each alignment (Fig. 1E). Further information about each result is displayed in the result table (Fig. 1F).

The results visualization and table provide the users with information so they can fill out the annotation form (Fig. 1D). The annotation form provides fields for the Annotation label, the Flag, Public notes, Private notes, and the Assigned to field. The Flag field provides a way for users to sort annotations into groups. Annotations flagged with a color will appear that color in the genome visualization. Color flags have no predefined meaning; therefore what each color represents can be determined by the user/lab. There are also several predefined flags such as the 'UNANNOTATED' flag, which is assigned to newly created CDS annotations and the 'REVIEW NAME' flag, which is available to signal that the annotation needs to be revisited. The notes fields provide a way for users to describe their findings in greater detail. Public notes will be shown in the GenBank file created from the 'Export genome data' button, while private notes will not. The Assigned to field allows for adding the annotation to a user queue so it will show up in that user's assignment navigator.

\section{Tools and databases}

There are currently three homology search tools implemented in MAS: BLASTp, rpsblast (Reverse PSIBLAST), and HHsearch. BLASTp allows users to search for primary protein structure homology [21]. MAS includes three blastp databases by default: SWISS-PROT, NCBI's non-redundant (nr) protein database, and an internally generated database. SWISS-PROT is a manually curated database which strives to achieve high levels of annotation and minimal redundancy [22]. SWISS-PROT is a relatively small database but it contains highly accurate information. The nr protein database is massive and contains various levels of curated data from multiple sources such as RefSeq, UniProtKB, Protein Data Bank (PDB), Protein Research Foundation (PRF), and CDS translations from all proteins in GenBank [23]. The internal blast database is a database automatically generated by MAS. It includes the protein translations for all CDS annotations within the local MAS installation. It automatically updates itself when an annotation is edited or when a new genome is uploaded and supports consistency and self-review in the manual annotation process.

MAS uses rpsblast to search for homologous domains [21]. Rpsblast can be used to search against the Conserved Domain Database (CDD) [24]. The CDD contains protein domain models from SMART [25], Pfam [26], TIGRFAMS [27], COG [28], ProtClustDB [29], NCBIfam [30], and CDD's own curation efforts. It currently contains over fifty-five thousand protein and proteindomain models.

HHsearch allows users to search for distantly homologous proteins by taking a multiple sequence alignment (MSA) as input and searching a database of hidden Markov models (HMMs) [31]. MAS is able to use HHsearch to search for homology against a specially compiled version of PDB. PDB contains atomic level structures of proteins, DNA, and RNA. These structures have been experimentally derived from X-ray crystallography, nuclear magnetic resonance spectroscopy, or threedimensional electron microscopy. It currently holds over 155,000 models [32]. MAS uses a version of PDB, released by the maintainers of $\mathrm{HH}$-Suite, that is clustered to $70 \%$ maximum pairwise sequence identity. MAS uses HHblits to create the MSA used as input into HHsearch. HHblits builds an MSA from the query sequence by iteratively searching the Uniclust30 database. Uniclust30 contains UniProtKB sequences clustered at 30\% pairwise sequence identity [33].

\section{Additional capabilities}

Users can view a list of previously uploaded genomes or annotations through the genome list (Fig. 2) and annotation list tabs. Both these lists can be filtered with a search query and ordering can be changed based on selected columns. Relevant information and links to relevant pages for each genome/annotation are presented in tabular format. The annotation table provides a dropdown pane to present the annotation's sequence in FASTA format.

Each genome in MAS has its own details page which displays summary information and lists of all features and annotations found within that genome. From here, users may download a FASTA file for the genome or use the 'export genome data' option to download a tarball containing a FASTA file for the genome sequence, a multi-FASTA file containing translations of the genome's CDSs, a general feature format (GFF) file describing the genome's features, and an excel document listing the genome's features and annotations.

MAS provides a way for users to easily upload annotations produced outside of MAS through the 'upload annotations from excel' tab. The label, notes fields, and 


\begin{tabular}{|c|c|c|c|c|c|c|c|c|c|c|c|c|}
\hline \multicolumn{13}{|l|}{ Genomes } \\
\hline Show $10 \vee$ entries & & & & & & & & & & Search: & & \\
\hline Genome Name & Organism & $\begin{array}{l}\text { Genome } \\
\text { Length }\end{array}$ & $\stackrel{\#}{\operatorname{CDS}}$ & $\begin{array}{l}\text { \# CDS } \\
\text { Unannotated }\end{array}$ & $\begin{array}{l}\text { \# Needs } \\
\text { Review }\end{array}$ & $\begin{array}{l}\text { \# Green } \\
\text { Flag }\end{array}$ & $\begin{array}{l}\text { \# Yellow } \\
\text { Flag }\end{array}$ & $\begin{array}{l}\text { \# Red } \\
\text { Flag }\end{array}$ & $\begin{array}{l}\# \\
\text { Endolysin }\end{array}$ & $\begin{array}{l}\# \\
\text { tRNA }\end{array}$ & $\begin{array}{l}\text { Download } \\
\text { Sequence }\end{array}$ & Nav \\
\hline EX1_S_aureus_RSA050_Phi__133 & phage & 18056 & 22 & 0 & 0 & 20 & 0 & 0 & 2 & 0 & download fasta & is \\
\hline EX1_A_baumannii_4567_Phi_162 & phage & 92737 & 184 & 0 & 13 & 169 & 0 & 0 & 2 & 1 & download fasta & $\mathbb{B}$ \\
\hline EX1_A_baumannii_4567_Phi_163 & phage & 93448 & 181 & 0 & 13 & 166 & 0 & 0 & 2 & 0 & download fasta & $\mathbb{B}^{3}$ \\
\hline EX1_A_baumannii_103H_Phi_109 & phage & 164723 & 243 & 0 & 0 & 242 & 0 & 0 & 1 & 9 & download fasta & w \\
\hline EX2_A_baumanni__GR45_Phi_105 & phage & 164723 & 243 & 0 & 0 & 242 & 0 & 0 & 1 & 9 & download fasta & is \\
\hline EX2_E_coli_Phi_111 & phage & 166113 & 241 & 0 & 0 & 240 & 0 & 0 & 1 & 9 & download fasta & is \\
\hline EX2_E_coli_Phi_112 & phage & 166964 & 243 & 0 & 0 & 242 & 0 & 0 & 1 & 8 & download fasta & 10 \\
\hline EX2_E_coli_Phi_237 & phage & 43778 & 69 & 0 & 0 & 67 & 0 & 1 & 1 & 0 & download fasta & is \\
\hline EX2_K_pneumoniae_RS71_Phi_122A & A phage & 96468 & 198 & 198 & 0 & 0 & 0 & 0 & 0 & 1 & download fasta & w \\
\hline EX2_K_pneumoniae_RS71_Phi_122B & B phage & 46043 & 85 & 0 & 0 & 82 & 2 & 0 & 1 & 0 & download fasta & is \\
\hline Genome Name & Organism & $\begin{array}{l}\text { Genome } \\
\text { Length }\end{array}$ & $\ddot{\text { CDS }}$ & $\begin{array}{l}\text { \# CDS } \\
\text { Unannotated }\end{array}$ & $\begin{array}{l}\text { \# Needs } \\
\text { Review }\end{array}$ & $\begin{array}{l}\text { \# Green } \\
\text { Flag }\end{array}$ & $\begin{array}{l}\text { \# Yellow } \\
\text { Flag }\end{array}$ & $\begin{array}{l}\text { \# Red } \\
\text { Flag }\end{array}$ & $\begin{array}{l}\# \\
\text { Endolysin }\end{array}$ & $\begin{array}{l}\# \\
\text { tRNA }\end{array}$ & $\begin{array}{l}\text { Download } \\
\text { Sequence }\end{array}$ & Nav \\
\hline Showing 1 to 10 of 4720 entries & & & & & & & & Previous & 12 & 4 & $\begin{array}{ll}\ldots & 472\end{array}$ & Next \\
\hline
\end{tabular}

Fig. 2 The genome list. This list displays information about all genomes that have been uploaded to MAS. From this page, users can navigate to the details page of any genome, navigate to the result viewer for any genome, filter the data displayed with the search bar, download genome sequences as a FASTA file, and sort by column. This also provides a way of quickly identifying which annotation flags each genome contains

flag described in each row of the uploaded excel file will be matched to an existing annotation in the database by protein sequence.

\section{Usage example}

In this section, we illustrate how a general user could leverage the capabilities of MAS for their project by walking through an example use case. MAS was originally created to support bacteriophage genome annotation but has been extended for use in other organisms. In this example, we show how MAS can be used to characterize the genomes of bacteriophages which are candidates for use in phage therapy, i.e. phages which may be used to treat antibiotic resistant bacterial infections.

\section{Motivation}

It is important to have a large and diverse set of phages available in order to effectively treat a wide range of bacterial infections because phages are specific to their bacterial host. Subsequently, this raises a safety concern and it is likely not feasible to perform in vitro safety tests on all phages. Genomic sequencing and characterization offers a more achievable alternative to in vitro tests. Confidence in the safety of a phage can be strengthened by screening its genome for genes with potentially harmful functions. In this case, genes with harmful functions would include genes that increase bacterial virulence, antibiotic resistance (AR) genes, and genes which indicate a lysogenic lifestyle. The use of MAS would be beneficial in this situation for a variety of reasons:
- MAS excels at the annotation of novel genomes. Phage genomes are extremely diverse and many newly sequenced phages are highly novel. This means that the functions of genes within the genome will have to be predicted based on distant homology to previously characterized genes. Many sequence homology tools and automated annotation tools are not sensitive enough to detect distant homology. The HH-suite of tools, which contains HHsearch, was built to detect distant homology. To our knowledge, MAS is the only annotation editing tool which contains the tool HHsearch.

- MAS can annotate genomes with a high degree of accuracy. In this situation, the accuracy of annotations is critical because failure to detect a problematic gene could adversely affect a patient's health outcome or response to treatment. Annotations based on a single database are highly sensitive to, or biased by, poorly annotated sequences contained within that database. MAS allows users to compare homology search results across different tools and databases, giving users a better chance at detecting erroneous results.

- MAS can assist with the categorization of annotations. In this example, the user will make a determination on whether a gene is problematic or not during the annotation process. MAS can help users make this determination by providing evidence and by allowing them to assign annotations to categories through annotation flags. 
The same reasons for using MAS apply to other situations where annotation accuracy is critical, the genome is novel, or it is necessary to categorize a diverse set of genes.

\section{Preparing data for MAS}

To start using MAS, the user must first generate and prepare a genome sequence. This genome sequence can be created from raw sequencing data through de novo assembly or reference-based approaches [34]. Ideally, the user will generate a complete genome sequence or chromosome. Doing so may require the use of genome finishing techniques [35]. With respect to phage genomes, this would entail removal of assembly artifacts and genomic termini resolution [20].

\section{Uploading the genome}

Once the user has a genome sequence in FASTA format, they can start using MAS to annotate it. Before annotation can occur, the genome must be uploaded to MAS. The user has a number of options available at this stage. In most situations, the user will opt to allow MAS to automatically determine the location of genes in the uploaded sequence. MAS accomplishes this using GLIMMER and tRNAscan-SE [1, 36]. The user also has the option to perform gene-calling outside of MAS using a different automated gene-caller or a tool such as DNA master to manually determine gene locations [37]. In the latter situation, the user will upload the FASTA file using the "Upload Custom Genome" option whereas in the former situation, the user will upload the FASTA file using the "Upload Phage Genome" option. The physical orientation of a phage's chromosome is dependent on its packaging strategy [38], however, for many species of phages, this orientation is random between individuals. If this is the case, the user will allow MAS to automatically reorient the uploaded genome sequence to start from the terminase gene. This will facilitate comparative analysis between similar genomes. In other cases, the phage genome is flanked by direct repeat sequences. The user will provide the length of these sequences during genome sequence upload in order to allow MAS to annotate them automatically. The user also must select a name for the genome during the upload process. When naming a phage genome in MAS, the selected name must be unique and it is best practice to include information about the host bacterium. The user may also wish to include additional information in the genome name such as a project identifier, the sample source location, or the name of the laboratory that collected/sequenced the sample. Including this information in the genome name allows the user to query genomes based on it.

\section{Annotation}

To begin the annotation process, the user selects the homology search tools and databases they would like to use and initiates the searches for each CDS. If not limited by computational resources, the user may initiate searches for all available tools and databases. The searches will execute in the background and as soon as each individual search finishes, results will populate for the user to review. As results are becoming available, the user will navigate through the CDS in the uploaded genome, viewing the returned results for one at a time. As described in the previous Tools and Databases section, the user will have access to results from three homology search tools: BLASTp, HHSearch, and RPS-BLAST. HHsearch will be used to search the PDB, RPS-BLAST will be used to search against the CDD, and BLASTp will be performed against three separate databases: SWISS-PROT, NCBI's nt, and an internally generated BLAST database. The results for all of these tools will be accessible through a single webpage. The user will review these results to determine a name for the annotation, what notes to include, and what flag to assign to it. There are multiple things to consider in order to assign the best possible annotation. The user must consider which tools and databases returned results, the significance of the results, length of the result sequence, consistency among results, length of the alignment, and position of the alignment with respect to both the query sequence and the result sequence. In general, a good strategy is to quickly run-through the results from each database to see which tools returned statistically significant results. Once the user knows which results to consider, they can interrogate the results further and compare results across databases. The process of determining the best label for an annotation through the interrogation of results in MAS is summarized in Table 1.

In addition to the annotation's label, the user will decide which flag to assign to the annotation. The Flag field provides the user with a way of categorizing annotations. Some of the provided flag options have defined meanings. For example, the 'review name' flag signifies that the annotation needs further review and the 'tRNA' flag is automatically assigned to tRNA genes discovered by tRNAscan-SE. The user can also assign the annotation to a color using the flag field. The meaning of each color is left to the user's discretion. In our example, the user could assign color flags as a way of categorizing proteins according to risk. Proteins that would not adversely affect the efficacy or safety of phage therapy would be assigned the 'green' flag, while proteins which would have an adverse effect, such as an integrase gene or a toxin, would be assigned the 'red' flag. The color of each flag is reflected in the genome visualization, users can search for annotations by their flag, and genomes 


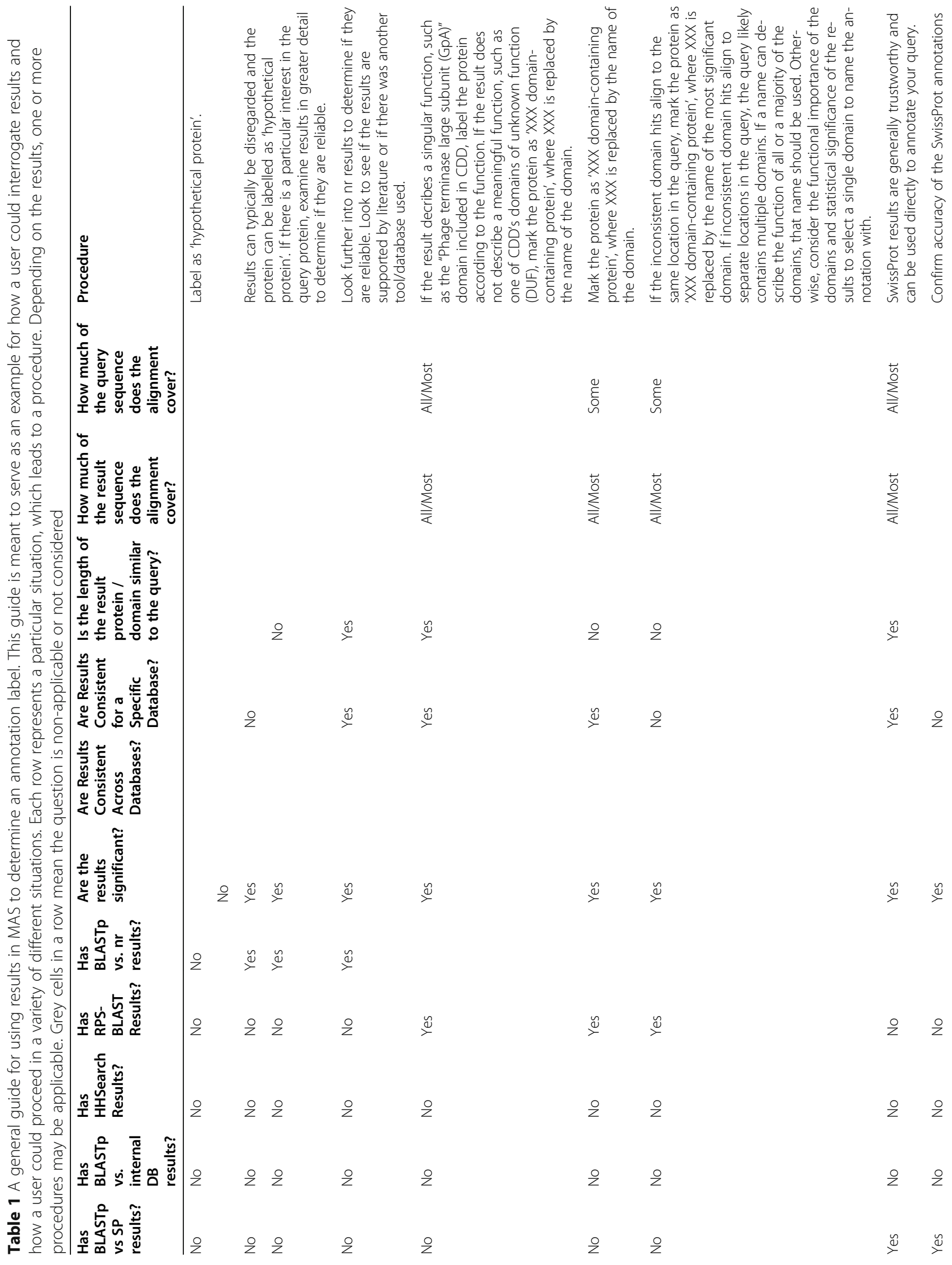




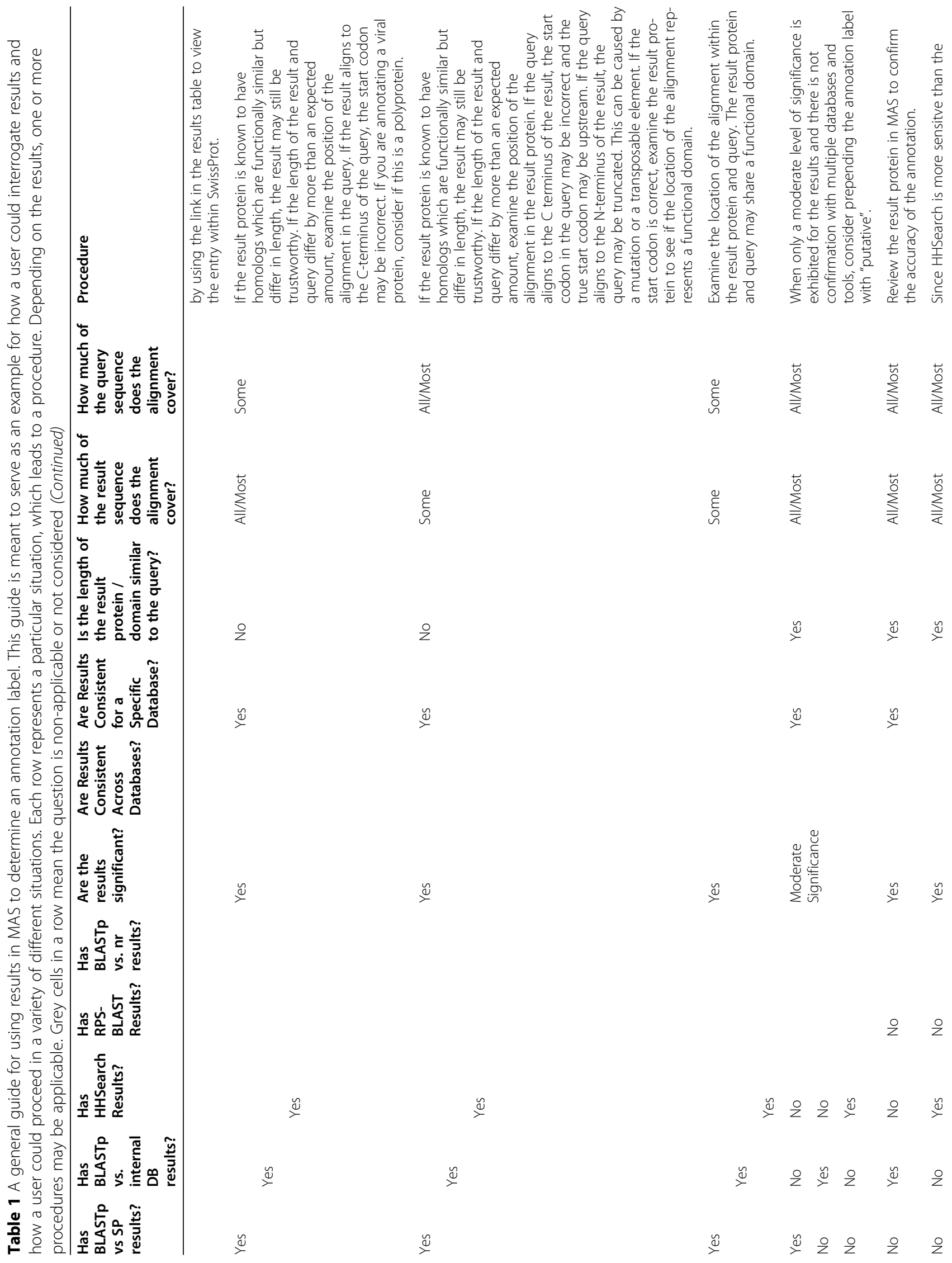




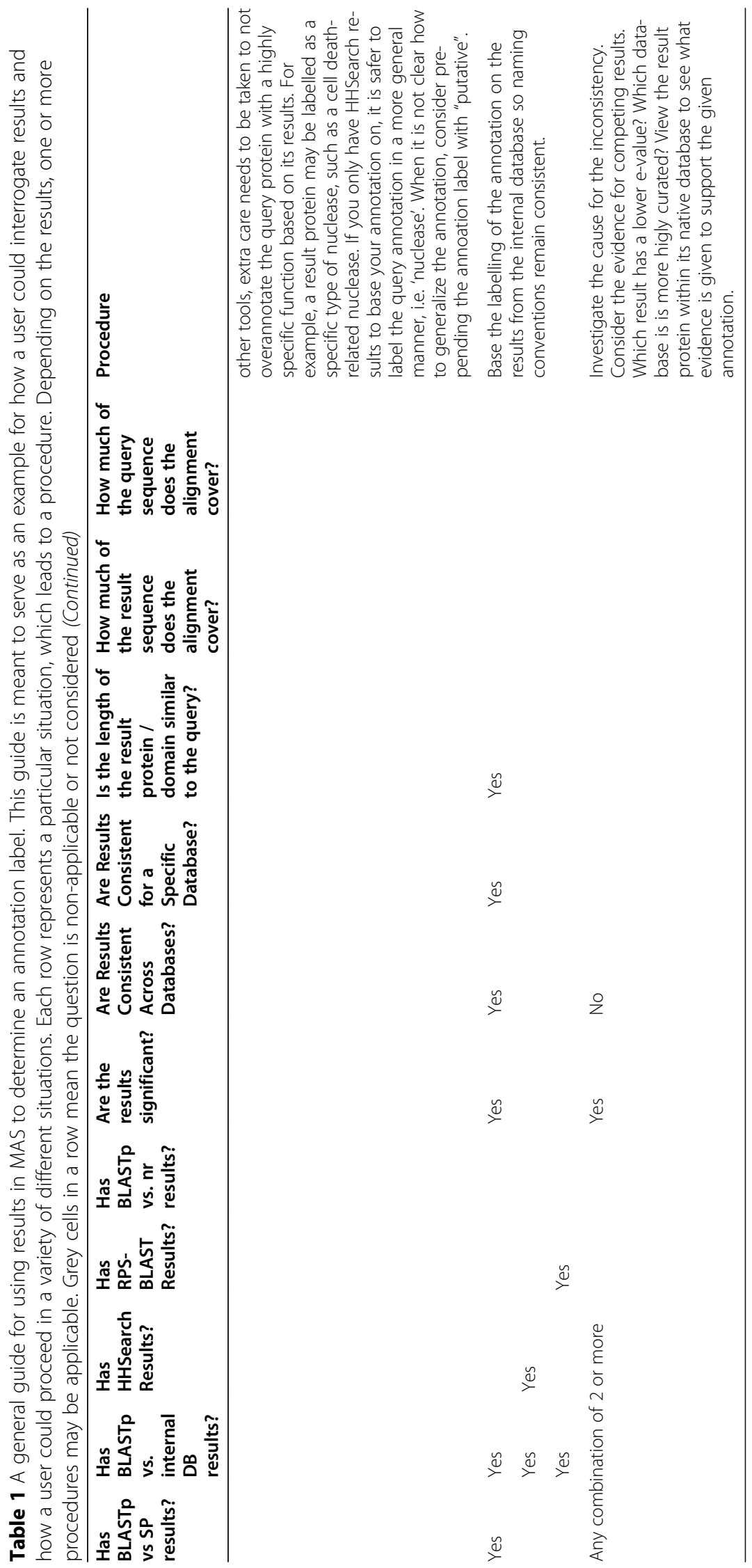


can be sorted according to the number of proteins assigned to each flag. In addition, each protein's flag will be saved in the internal BLAST database, aiding future annotation. Any important information that is not described by the annotation label or flag can be added to the notes fields. When the user finishes annotating a phage genome, the can export the phage's data in multiple formats using the "download deliverables" option on the phage details page.

\section{Future improvements}

MAS will serve as a platform for further development, with additional tools, databases, and features added to support the varying needs of its users. Planned future improvements include additional gene callers, improved genome visualizations, transmembrane domain prediction, rRNA prediction, additional feature types, custom flags, and support for annotating alternative splicing and polyproteins.

\section{Implementation}

\section{General overview}

MAS was designed to run as a local web-server allowing multiple users to simultaneously interact through a webbrowser and to collaborate by accessing and updating shared data. The annotation process starts when a user uploads an unannotated genome in a FASTA formatted file (Fig. 3). The user can also provide the coordinates of coding sequences (CDS) within the genome, or MAS can automatically predict their locations. The genome and features (such as coding sequences) are stored in a database. The user can then initiate a variety of homology searches using the graphical user interface (GUI). Homology search results are also stored by MAS and are presented to the user in a graphical representation and in a result table. The user can then examine these results to determine how to annotate the associated protein. Annotation information is provided to MAS through a form and is saved to the database upon submission. Users can navigate information stored in the database

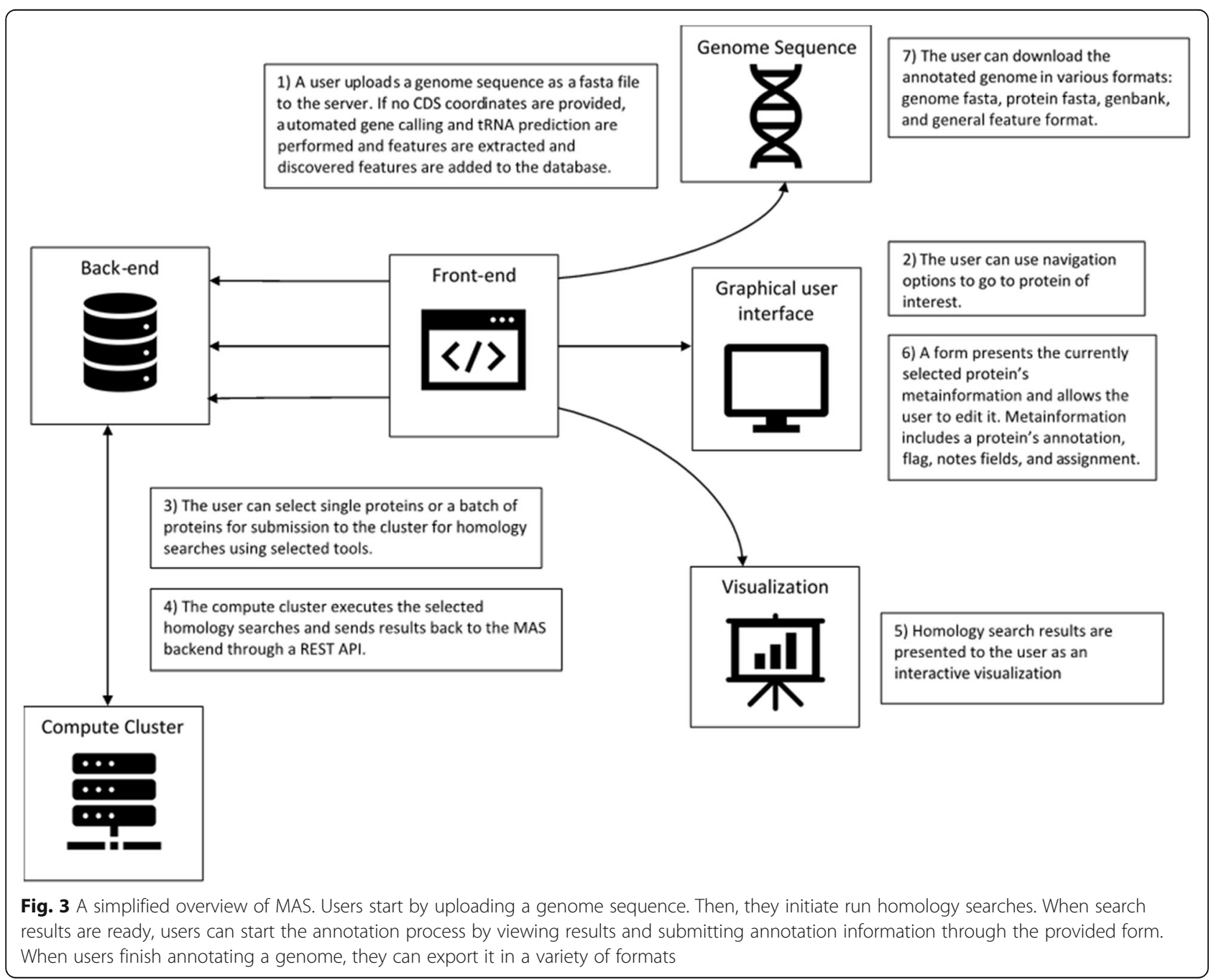


through a variety of views and information can be exported from the database in a variety of formats.

\section{Architecture}

MAS consists of multiple interacting components which work together to provide a functional webserver (Supplementary Figure 1). Most of these components are containerized using Docker. MAS uses Docker to make installation and updates easier for users and to provide enhanced portability. There are three Docker containers that are built and run through the docker-compose command: a container housing the Apache server, a container housing the SQL server, and a container for the message broker. The fourth and final component of the MAS system is the worker, which is installed directly on the host machine and is responsible for handling computationally intensive tasks.

The MAS server uses the Django (v3.1) web framework and is written in Python (v3.8), JavaScript, and HTML. The Django/Apache server container is built from a Dockerfile using the official Docker Apache httpd image as a base image. Interactive visualizations are created within JavaScript using the D3.js library. The Biopython python library [39] is used to parse FASTA files and results from homology searches. The homology search pipeline uses the Luigi framework in order to help manage long running batch processes. With a default configuration, the MAS website uses port 8080 and the Luigi scheduler uses port 8082 . Homology search results are stored as text files by the server to a Docker volume.

Uploaded genomes, genomic features, annotations, homology search result metadata, and user information are stored within a MariaDB SQL server (Supplementary Figure 2). The SQL server resides in a Docker container built from the official MariaDB Docker image. Data stored to the database is written to a Docker volume, which allows the data to persist when the SQL server container is destroyed or rebuilt.

MAS allows users to trigger long running homology search pipelines. These pipelines are activated through the Celery distributed task queue allowing them to be executed asynchronously by the worker, outside of the HTTP request/response cycle. The MAS server communicates tasks which need to be executed to the worker through a RabbitMQ message broker. The worker can then directly execute the task, or if the task involves a pipeline of multiple long-running batch process, it can initiate a Luigi pipeline. Luigi pipelines are initiated by the worker when users submit homology searches to the server. These pipelines can execute searches directly on the host machine or in a high-performance computing (HPC) environment through SGE or SLURM batchqueuing systems depending on how MAS was configured during the installation process. Homology search pipelines communicate results back to the server through a REST API. The worker is installed directly on the host machine, rather than in a Docker container, in order to enable cluster compatibility.

\section{Conclusion}

MAS provides researchers a way to streamline the process of manual functional annotation. Running homology searches in MAS requires less overhead than running the searches through online services and less bioinformatics expertise than running searches through the command-line. MAS facilitates reproducibility by saving search results, annotations, and annotation history in a structured database. Team annotation efforts benefit from the user management system MAS provides and from its ability to allow users to view and edit shared data concurrently. We will continue to work towards integration of additional tools and databases into MAS in order to provide annotators with additional information and visualizations to improve the quality of their annotations even further.

\section{Availability and requirements}

Project name: Manual Annotation Studio

Project home page: https://github.com/BDRDGenomics/MAS

Operating System(s): Server: Linux and OSX, User: Platform independent

Programming languages: Python, JavaScript, HTML, CSS

Other requirements: Docker, Conda package manager, modern web browser

License: GNU General Public License v3.0

Any restrictions to use by non-academics: None

\section{Abbreviations}

API: Application programming interface; CDD: Conserved Domain Database; CDS: Coding sequences; GFF: General Feature Format; GUI: Graphical User Interface; HMM: Hidden Markov Model; HPC: High-Performance Computing; LAN: Local Area Network; MAS: Manual Annotation Studio; NR: NonRedundant; PDB: Protein Data Bank; PGAP: Prokaryotic Genome Annotation Pipeline; PRF: Protein Research Foundation; RAST: Rapid Annotation using Subsystems Technology; SLURM: Simple Linux Utility for Resource Management

\section{Supplementary Information}

The online version contains supplementary material available at https://doi. org/10.1186/s12864-021-08029-8.

Additional file 1: Supplementary Figure 1. The architecture of MAS. Installation of MAS will automatically build three Docker containers: 1) a Docker container containing MAS's code, the Apache server, and the Luigi daemon, 2) a Docker container containing the database server, and 3) a Docker container containing the message broker used to send tasks to the MAS Worker. These three Docker containers exist under the same Docker engine and communicate with each other through a bridge network. Computationally intensive jobs are sent to the MAS worker 
using the Celery distributed task queue. The worker can execute the workload on the host machine or on a compute cluster through either the SLURM or SGE batch-queuing systems.

Additional file 2: Supplementary Figure 2. MAS Database Schema.

\section{Acknowledgements}

Not applicable.

\section{Disclaimer}

The views, opinions, interpretations, conclusions, and recommendations expressed in this article reflect the results of research conducted by the authors and do not necessarily reflect the official policy or position of the Department of the Navy, Department of Defense, nor the United States Government.

KBL and RZC are federal employees of the United States government. This work was prepared as part of their official duties. Title 17 U.S.C. 105 provides that 'copyright protection under this title is not available for any work of the United States Government.' Title 17 U.S.C. 101 defines a U.S. Government work as work prepared by a military service member or employee of the U.S. Government as part of that person's official duties.

\section{Authors' contributions}

MRL developed the project concept, led software development, and wrote the manuscript. MP was a major contributor to software development. LVV contributed to software development, reviewed the manuscript, and helped determine the tools and databases included in MAS. KAL reviewed the manuscript, helped determine software parameters, and was a primary software tester. GKR reviewed the manuscript and served as a resource for technical troubleshooting. RZC reviewed the manuscript and served as the technical project manager. KBL secured project funding, provided management oversight, and reviewed the manuscript. The author(s) read and approved the final manuscript.

\section{Funding}

This work was supported by WUNA1417, the Naval Research Enterprise Internship Program (NREIP), and by the Assistant Secretary of Defense for Health Affairs, through the Peer Reviewed Medical Research Program Focused Program Award, Log Number PR182667.

\section{Availability of data and materials}

MAS source code is available on GitHub, https://github.com/BDRDGenomics/MAS

\section{Declarations}

Ethics approval and consent to participate

Not applicable.

\section{Consent for publication}

Not applicable.

\section{Competing interests}

The authors declare that they have no competing interests.

\section{Author details}

${ }^{1}$ Genomics and Bioinformatics Department, Biological Defense Research Directorate, Naval Medical Research Center, Fort Detrick, MD, USA. ${ }^{2}$ Leidos, Reston, VA, USA. ${ }^{3}$ Mount St. Mary's University, Emmitsburg, MD, USA.

Received: 23 March 2021 Accepted: 22 September 2021

Published online: 09 October 2021

\section{References}

1. Delcher AL, Bratke KA, Powers EC, Salzberg SL. Identifying bacterial genes and endosymbiont DNA with Glimmer. Bioinformatics. 2007;23(6):673-9. https://doi.org/10.1093/bioinformatics/btm009.

2. Besemer J, Borodovsky M. GeneMark: web software for gene finding in prokaryotes, eukaryotes and viruses. Nucleic Acids Res. 2005;33(Web Server issue):W451-4
3. Hyatt D, Chen GL, Locascio PF, Land ML, Larimer FW, Hauser LJ. Prodigal: prokaryotic gene recognition and translation initiation site identification. BMC Bioinformatics. 2010;11(1):119. https://doi.org/10.1186/1471-2105-11-11 9.

4. Steward CA, Parker APJ, Minassian BA, Sisodiya SM, Frankish A, Harrow J. Genome annotation for clinical genomic diagnostics: strengths and weaknesses. Genome Med. 2017;9(1):49. https://doi.org/10.1186/s13073-0170441-1.

5. Baric RS, Crosson S, Damania B, Miller SI, Rubin EJ. Next-Generation HighThroughput Functional Annotation of Microbial Genomes. mBio. 2016;7(5): e01245-16.

6. Mills R, Rozanov M, Lomsadze A, Tatusova T, Borodovsky M. Improving gene annotation of complete viral genomes. Nucleic Acids Res. 2003;31(23):704155. https://doi.org/10.1093/nar/gkg878.

7. Overbeek R, Olson R, Pusch GD, Olsen GJ, Davis JJ, Disz T, et al. The SEED and the rapid annotation of microbial genomes using subsystems technology (RAST). Nucleic Acids Res. 2014;42(Database issue):D206-14. https://doi.org/10.1093/nar/gkt1226

8. Seemann T. Prokka: rapid prokaryotic genome annotation. Bioinformatics. 2014;30(14):2068-9. https://doi.org/10.1093/bioinformatics/btu153.

9. Tatusova T, DiCuccio M, Badretdin A, Chetvernin V, Nawrocki EP, Zaslavsky L, et al. NCBI prokaryotic genome annotation pipeline. Nucleic Acids Res. 2016; 44(14):6614-24. https://doi.org/10.1093/nar/gkw569.

10. Ejigu GF, Jung J. Review on the Computational Genome Annotation of Sequences Obtained by Next-Generation Sequencing. Biology (Basel). 2020; 9(9):295.

11. Dominguez Del Angel V, Hjerde E, Sterck L, Capella-Gutierrez S, Notredame C, Vinnere Pettersson O, et al. Ten steps to get started in Genome Assembly and Annotation. F1000Res. 2018;7:ELIXIR-148.

12. Naveed M, Tehreem S, Usman M, Chaudhry Z, Abbas G. Structural and functional annotation of hypothetical proteins of human adenovirus: prioritizing the novel drug targets. BMC Res Notes. 2017;10(1):706. https:// doi.org/10.1186/s13104-017-2992-z.

13. Sen T, Verma NK. Functional Annotation and Curation of Hypothetica Proteins Present in A Newly Emerged Serotype 1c of Shigella flexneri: Emphasis on Selecting Targets for Virulence and Vaccine Design Studies. Genes (Basel). 2020;11(3):340.

14. Kumar S. Structure and functional annotation of hypothetical proteins having putative rubisco activase function from Vitis vinifera. Bioinformation. 2015;11(1):11-6. https://doi.org/10.6026/97320630011011.

15. Chavez-Fumagalli MA, Schneider MS, Lage DP, Machado-de-Avila RA, Coelho EA. An in silico functional annotation and screening of potential drug targets derived from Leishmania spp. hypothetical proteins identified by immunoproteomics. Exp Parasitol. 2017;176:66-74. https://doi.org/10.101 6/j.exppara.2017.03.005.

16. Meyer F, Goesmann A, McHardy AC, Bartels D, Bekel T, Clausen J, et al. GenDB--an open source genome annotation system for prokaryote genomes. Nucleic Acids Res. 2003;31(8):2187-95. https://doi.org/10.1093/na r/gkg312.

17. Dunn NA, Unni DR, Diesh C, Munoz-Torres M, Harris NL, Yao E, et al. Apollo: Democratizing genome annotation. PLoS Comput Biol. 2019;15(2):e1006790. https://doi.org/10.1371/journal.pcbi.1006790.

18. Lawrence J. DNA Master. 5.0.2 ed: University of Pittsburgh; 2007.

19. Salisbury A, Tsourkas PK. A Method for Improving the Accuracy and Efficiency of Bacteriophage Genome Annotation. Int J Mol Sci. 2019;20(14):3391.

20. Philipson CW, Voegtly LJ, Lueder MR, Long KA, Rice GK, Frey KG, et al. Characterizing Phage Genomes for Therapeutic Applications. Viruses. 2018; 10(4):188

21. Camacho C, Coulouris G, Avagyan V, Ma N, Papadopoulos J, Bealer K, et al. BLAST+: architecture and applications. BMC Bioinformatics. 2009;10(1):421. https://doi.org/10.1186/1471-2105-10-421.

22. Bairoch A, Apweiler R. The SWISS-PROT protein sequence database and its supplement TrEMBL in 2000. Nucleic Acids Res. 2000;28(1):45-8. https://doi. org/10.1093/nar/28.1.45

23. Coordinators NR. Database resources of the National Center for biotechnology information. Nucleic Acids Res. 2016;44(D1):D7-19. https:// doi.org/10.1093/nar/gkv1290

24. Lu S, Wang J, Chitsaz F, Derbyshire MK, Geer RC, Gonzales NR, et al. CDD/ SPARCLE: the conserved domain database in 2020. Nucleic Acids Res. 2020; 48(D1):D265-D8, https://doi.org/10.1093/nar/gkz991. 
25. Letunic I, Bork P. 20 years of the SMART protein domain annotation resource. Nucleic Acids Res. 2018;46(D1):D493-D6. https://doi.org/10.1093/ nar/gkx922.

26. El-Gebali S, Mistry J, Bateman A, Eddy SR, Luciani A, Potter SC, et al. The Pfam protein families database in 2019. Nucleic Acids Res. 2019;47(D1): D427-D32. https://doi.org/10.1093/nar/gky995.

27. Haft DH, Selengut JD, Richter RA, Harkins D, Basu MK, Beck E. TIGRFAMs and genome properties in 2013. Nucleic Acids Res. 2013;41(Database issue): D387-95. https://doi.org/10.1093/nar/gks1234.

28. Tatusov RL, Natale DA, Garkavtsev IV, Tatusova TA, Shankavaram UT, Rao BS, et al. The COG database: new developments in phylogenetic classification of proteins from complete genomes. Nucleic Acids Res. 2001;29(1):22-8. https://doi.org/10.1093/nar/29.1.22

29. Klimke W, Agarwala R, Badretdin A, Chetvernin S, Ciufo S, Fedorov B, et al. The National Center for biotechnology Information's protein clusters database. Nucleic Acids Res. 2009;37(Database issue):D216-23. https://doi. org/10.1093/nar/gkn734.

30. Haft DH, DiCuccio M, Badretdin A, Brover V, Chetvernin V, O'Neill K, et al. RefSeq: an update on prokaryotic genome annotation and curation. Nucleic Acids Res. 2018;46(D1):D851-D60. https://doi.org/10.1093/nar/gkx1068.

31. Steinegger M, Meier M, Mirdita M, Vohringer $H$, Haunsberger SJ, Soding J. $\mathrm{HH}$-suite3 for fast remote homology detection and deep protein annotation. BMC Bioinformatics. 2019;20(1):473. https://doi.org/10.1186/s12 859-019-3019-7.

32. Goodsell DS, Zardecki C, Di Costanzo L, Duarte JM, Hudson BP, Persikova I, et al. RCSB protein data Bank: enabling biomedical research and drug discovery. Protein Sci. 2020;29(1):52-65. https://doi.org/10.1002/pro.3730.

33. Mirdita M, von den Driesch L, Galiez C, Martin MJ, Soding J, Steinegger M. Uniclust databases of clustered and deeply annotated protein sequences and alignments. Nucleic Acids Res. 2017;45(D1):D170-D6. https://doi.org/1 0.1093/nar/gkw1081.

34. Flicek P, Birney E. Sense from sequence reads: methods for alignment and assembly. Nat Methods. 2009;6(11 Suppl):S6-S12. https://doi.org/10.1038/ nmeth.1376.

35. Nagarajan N, Cook C, Di Bonaventura M, Ge H, Richards A, Bishop-Lilly KA, et al. Finishing genomes with limited resources: lessons from an ensemble of microbial genomes. BMC Genomics. 2010;11(1):242. https://doi.org/10.11 86/1471-2164-11-242.

36. Chan PP, Lowe TM. tRNAscan-SE: searching for tRNA genes in genomic sequences. Methods Mol Biol. 1962;2019:1-14. https://doi.org/10.1007/ 978-1-4939-9173-0_1.

37. Pope WH, Jacobs-Sera D. Annotation of bacteriophage genome sequences using DNA master: an overview. Methods Mol Biol. 1681;2018:217-29. https://doi.org/10.1007/978-1-4939-7343-9_16.

38. Merrill BD, Ward AT, Grose JH, Hope S. Software-based analysis of bacteriophage genomes, physical ends, and packaging strategies. BMC Genomics. 2016;17(1):679. https://doi.org/10.1186/s12864-016-3018-2.

39. Cock PJ, Antao T, Chang JT, Chapman BA, Cox CJ, Dalke A, et al. Biopython: freely available Python tools for computational molecular biology and bioinformatics. Bioinformatics. 2009;25(11):1422-3. https://doi.org/10.1093/ bioinformatics/btp163.

\section{Publisher's Note}

Springer Nature remains neutral with regard to jurisdictional claims in published maps and institutional affiliations.

Ready to submit your research? Choose BMC and benefit from:

- fast, convenient online submission

- thorough peer review by experienced researchers in your field

- rapid publication on acceptance

- support for research data, including large and complex data types

- gold Open Access which fosters wider collaboration and increased citations

- maximum visibility for your research: over $100 \mathrm{M}$ website views per year

At BMC, research is always in progress.

Learn more biomedcentral.com/submissions 